\title{
Atorvastatin partially inhibits the epithelial-mesenchymal transition in A549 cells induced by TGF- $\beta 1$ by attenuating the upregulation of SphK1
}

\author{
ZHIQIANG FAN $^{1 *}$, HANDONG JIANG $^{2 *}$, ZILI WANG $^{3}$ and JIEMING QU ${ }^{4}$ \\ ${ }^{1}$ Department of Pulmonary Medicine, Huadong Hospital, Shanghai Medical College, Fudan University, Shanghai 200040; \\ ${ }^{2}$ Department of Pulmonary Medicine, Renji Hospital, Shanghai Jiao Tong University School of Medicine, Shanghai 200127; \\ ${ }^{3}$ Department of Chemotherapy, The First Affiliated Hospital of Fujian Medical University, Fuzhou 350005; \\ ${ }^{4}$ Department of Pulmonary Medicine, Ruijin Hospital, Shanghai Jiaotong University \\ School of Medicine, Shanghai 200025, P.R. China
}

Received February 6, 2016; Accepted March 17, 2016

DOI: $10.3892 /$ or.2016.4897

\begin{abstract}
Statins are the most effective drugs used in the reduction of intracellular synthesis of cholesterol. Numerous studies have confirmed that statins reduce the risk of multiple types of cancers. Statin use in cancer patients is associated with reduced cancer-related mortality. Epithelial-to-mesenchymal transition (EMT), a complicated process programmed by multiple genes, is an important mechanism of cancer metastasis. We explored the effect and mechanism of atorvastatin on the EMT process in A549 cells by establishing an EMT model in vitro induced by TGF- $\beta 1$, and evaluated the effects of atorvastatin on the lower signaling pathway of TGF- $\beta 1$ stimulation. Our results showed that atorvastatin partially inhibited the EMT process, and inhibited cell migration and actin filament remodeling. Transcriptional upregulation of ZEB1 and protein sphingosine kinase 1 (SphK1) induced by TGF- $\beta 1$ was also suppressed. SphK1 plasmid transient transfection strengthened the EMT process induced by TGF- $\beta 1$ in the presence of atorvastatin. Our experiments confirmed that atorvastatin can partially inhibit the EMT process of non-small cell lung cancer cells induced by TGF- $\beta 1$ by attenuating the upregulation of SphK1.
\end{abstract}

\section{Introduction}

Lung cancer is the leading cause of cancer-related deaths worldwide. Approximately $85 \%$ of all lung cancers are non-small

Correspondence to: Dr Jieming Qu, Department of Pulmonary Medicine, Ruijin Hospital, Shanghai Jiaotong University School of Medicine, 197 Ruijin 2nd Road, Shanghai 200025, P.R. China

E-mail: jmqu0906@163.com

${ }^{*}$ Contributed equally

Key words: lung cancer, epithelial-mesenchymal transition, atorvastatin, SphK1, TGF- $\beta 1$ cell lung cancer (NSCLC). Despite advances in early detection and standard treatments, NSCLC is often diagnosed at an advanced stage and has a poor prognosis (1). Metastases are the cause of $90 \%$ of human cancer-related deaths (2).

Epithelial-to-mesenchymal transition (EMT) is a cellular process during which epithelial cells lose their polarized organization and cell-cell junctions, undergo changes in cell shape and in cytoskeletal organization and acquire mesenchymal characteristics, such as fibroblast-like cell morphology and increased cell migration and invasion (3). Transcriptional factors which are essential for EMT, such as Snail, Slug and ZEB1 are induced by growth factors in most cases. The endogenous presence or the forced expression of these transcriptional factors in cancer cells have been linked with the loss of E-cadherin and the gain of vimentin (4). EMT endows cells with migratory and invasive properties, induces stem cell properties, prevents apoptosis and senescence and contributes to immunosuppression (5).

Statins, which inhibit the 3-hydroxy-3-methylglutarylcoenzyme A reductase (HMG-CoAR) enzyme, are the most effective drugs used in the reduction of intracellular synthesis of cholesterol (6). The interactions between statins and HMG-CoA reductase prevent the conversion of $\mathrm{HMG}-\mathrm{CoA}$ to L-mevalonate resulting in the inhibition of downstream cholesterol biosynthesis, and numerous isoprenoid metabolites such as geranylgeranyl pyrophosphate (GGPP) and farnesyl pyrophosphate (FPP) (7). The isoprenoids are lipid moieties that are added to various proteins, including G-proteins and the G-protein subunits RAS, Rho, Rac and Cdc42, during post-translational modification (prenylation) and anchor these proteins to the cell membrane (8). Prenylation also occurs in many cellular and systemic regulatory pathways that are partly responsible for the pleiotropic effects of statins (9). Other pleiotropic effects may be independent of prenylation or inhibition of cholesterol production such as cell cycle arrest (10). Numerous studies have confirmed that statins reduce the risk of multiple types of cancers (11-14). Statin use in cancer patients is also associated with reduced cancerrelated mortality (15). 
The sphingosine kinase 1 (SphK1) can convert sphingosine to sphingosine 1-phosphate (16), and can be upregulated by TGF- $\beta$ in fibroblasts and myofibroblasts (17). TGF- $\beta$ can upregluate SphK1 in A549 cells (18). All of these facts indicate that SphK1 has roles in the TGF- $\beta$ lower signaling pathway.

EMT, a complicated process programmed by many genes, is an important mechanism for cancer metastasis. In the present research, we evaluated the effect and mechanism of atorvastatin on the EMT process in NSCLC cells by establishing an EMT model in vitro induced by TGF- $\beta 1$, and we investigated the effects of atorvastatin on the lower signaling pathway of TGF- $\beta 1$ as well as its effect on SphK1.

\section{Materials and methods}

Cell culture and reagents. Human lung adenocarcinoma A549 and NCI-H1975 cells were obtained from the American Type Culture Collection (ATCC; Manassas, VA, USA). A549 cells were cultured in Dulbecco's modified Eagle's medium (DMEM) with $10 \%$ fetal bovine serum (FBS) (Gibco, Grand Island, NY, USA) at $37^{\circ} \mathrm{C}$ in a humidified $5 \% \mathrm{CO}_{2}$ atmosphere. NCI-H1975 cells were maintained in RPMI-1640 medium with $10 \%$ FBS. The cells were trypsinized and seeded in 12-well tissue culture plates, and were treated with atorvastatin (Sigma, St. Louis, MO, USA) or TGF- $\beta 1$ (PeproTech Inc., Rocky Hill, NJ, USA) for different time periods in serum-free medium. The rabbit anti-human antibodies, such as E-cadherin, phospho-Smad2 (S465/467), Smad2, phospho-Smad3 (Ser423/425), Smad3, phospho-AKT (S473), AKT, phospho-ERK (T202/Y204), ERK, Snail and Slug were purchased from Cell Signaling Technology (Danvers, MA, USA). The monoclonal mouse anti-human antibodies, such as vimentin, SphK1 and ZEB1 were purchased from Abcam (Cambridge, UK). The polyclonal rabbit anti-human $\beta$-actin antibody was purchased from Sigma-Aldrich. The monoclonal mouse anti-human glyceraldehyde-3-phosphate dehydrogenase (GAPDH) antibody, horseradish peroxidase-conjugated anti-mouse and anti-rabbit secondary antibodies were all purchased from KangChen Bio-tech (Shanghai, China). BCA protein assay kit was purchased from Beyotime Biotechnology (Shanghai, China). The pBABE-SphK1 plasmid was a kind gift synthesized by the Shanghai Institute of Materia Medica (Chinese Academy of Sciences).

Transwell assay. The migration assay was carried out using 8- $\mu \mathrm{m}$ pore size Transwell filters (6.5-mm diameter; Corning Inc., Corning, NY, USA). Following pretreatment with atorvastatin for $24 \mathrm{~h}$, the A549 cells were detached and single-cell suspensions were placed at $1 \times 10^{5}$ cells/well into the upper chamber in $0.1 \mathrm{ml}$ of serum-free medium. In the lower chamber, DMEM supplemented with $10 \%$ FBS was placed as a chemoattractant. Atorvastatin and TGF- $\beta 1$ were added at constant concentration to both the upper and the lower chambers. After $18 \mathrm{~h}$ of incubation in $5 \% \mathrm{CO}_{2}$ at $37^{\circ} \mathrm{C}$, the filters were fixed with $90 \%$ ethanol for $30 \mathrm{~min}$ and stained with crystal violet. Cells from the upper surface of the chamber were removed with gentle swabbing and the invasive cells on the lower surface of the filters were examined by bright field microscopy. The average number from five randomly chosen fields was counted.
Immunofluorescence analysis. Stock solutions of FITCphalloidin were made in DMSO at $0.2 \mathrm{mg} / \mathrm{ml}$. Final staining solutions in phosphate-buffered saline (PBS) were at the concentration of $5 \mu \mathrm{g} / \mathrm{ml}$. Monolayers of A549 cells were serum-starved and treated with atorvastatin on glass coverslips for $24 \mathrm{~h}$, and then treated with atorvastatin and TGF- $\beta 1$ for $24 \mathrm{~h}$. The A549 cells were washed with PBS and fixed for $10 \mathrm{~min}$ in $4 \%$ polyformaldehyde, and then extensively washed in PBS three times. After being permeabilized with $0.1 \%$ Triton X-100 in PBS and washed again in PBS, the A549 cells were stained with $5 \mu \mathrm{g} / \mathrm{ml}$ FITC-phalloidin. Cover slides were mounted onto slides with $1 \%$ 4'6-diamidine-2'-phenylindole dihydrochloride (DAPI). Cells were examined under an Olympus Fluoview FV1000 confocal microscope (Olympus, Tokyo, Japan).

Western blot analysis. The cells were harvested, rinsed with cold PBS and lysed in buffer supplemented with protease inhibitors for $1 \mathrm{~h}$ as previously described (19). The protein samples were separated by $10 \%$ sodium dodecyl sulfate-polyacrylamide gel electrophoresis and electrophoretically transferred to nitrocellulose. The nitrocellulose membrane was blocked with 5\% milk in Tris-buffered saline and Tween-20 (TBST) for $1 \mathrm{~h}$ at room temperature and incubated with primary antibodies at $4^{\circ} \mathrm{C}$ overnight. Then, the membrane was washed with TBST for 15 min each time for three times and incubated with secondary antibodies at room temperature for $1 \mathrm{~h}$. To ensure that equal amounts of sample protein were applied for electrophoresis and immunoblotting, GAPDH or $\beta$-actin was used as a protein loading control. All experiments were performed at least three independent times.

Transient transfection of the SphK1 plasmid. A549 cells were plated in 12-well plates in DMEM supplemented with 10\% FBS overnight to reach $\sim 80-90 \%$ confluency. Then, the cells were transfected with the pBABE-SphK1 plasmid and pBABE-puro using Lipofectamine 2000 reagent (Invitrogen, Carlsbad, CA, USA) according to the manufacturer's instructions and replaced with culture medium containing $1 \mu \mathrm{M}$ atorvastatin at $5 \mathrm{~h}$ after transfection. Twenty-four hours later, the cells were treated with atorvastatin and TGF- $\beta 1$ for another $24 \mathrm{~h}$. Then, the cells were harvested for protein quantitative analysis using the BCA protein assay kit and western blot analysis.

RNA extraction and real-time PCR for SphK1 mRNA expression. A549 cells were maintained in 12-well plates with serum-free medium and exposed to atorvastatin for $24 \mathrm{~h}$ before being treated with TGF- $\beta 1$ for $48 \mathrm{~h}$. Total RNA was extracted using Takara MiniBEST Universal RNA Extraction kit (Takara Bio, Dalian, China) according to the manufacturer's instructions. cDNA was synthesized with PrimeScript reverse transcriptase using oligo(dT) primer and $2 \mu \mathrm{g}$ of total RNA (Takara PrimeScript ${ }^{\mathrm{TM}}$ RT Master Mix) (both from Takara Bio). Real-time PCR assays were conducted using SYBR Premix Ex Taq ${ }^{\mathrm{TM}}$ according to the manufacturer's instructions (Takara Bio). Amplification was performed in $20 \mu 1$ reactions $\left(0.4 \mu 1\right.$ primer, $10 \mu \mathrm{l}$ SYBR Premix Ex Taq $\left.{ }^{\mathrm{TM}}\right)$ containing $2 \mu \mathrm{l}$ of cDNA in 40 cycles at $95^{\circ} \mathrm{C}(3 \mathrm{sec})$ and $60^{\circ} \mathrm{C}$ (30 sec) following pre-denaturation at $95^{\circ} \mathrm{C}(30 \mathrm{sec})$. Data normalization was performed using GAPDH as a reference 


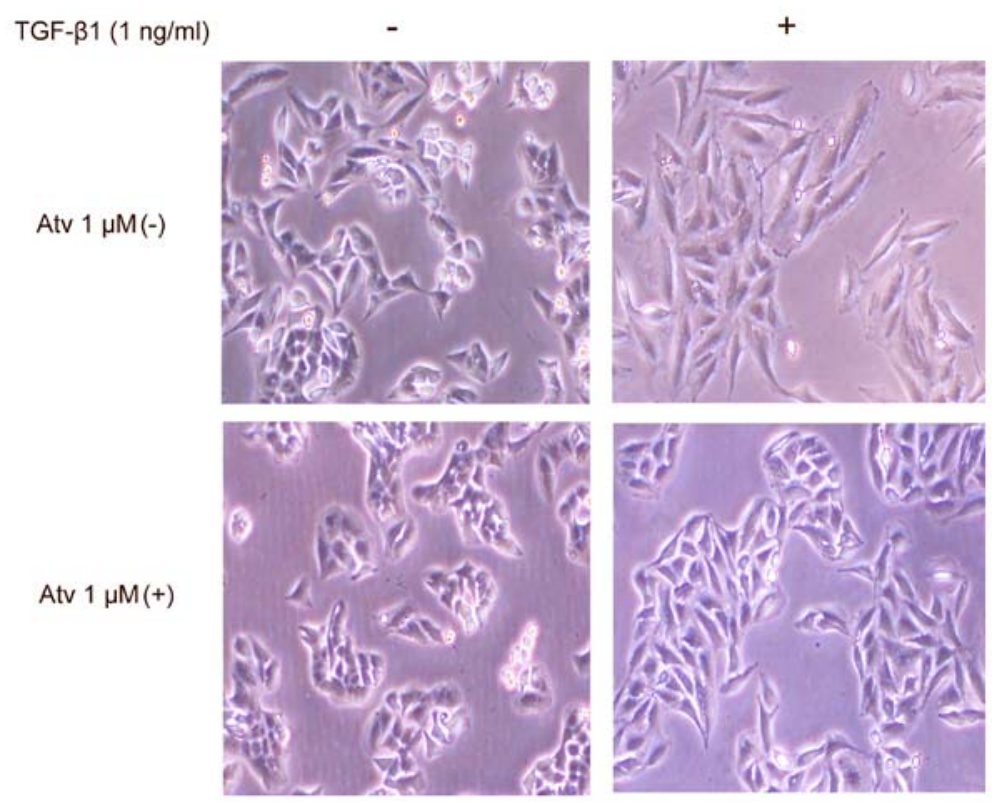

Figure 1. A549 cells display complete transformation to fibroblast-like characteristics when exposed to TGF- $\beta 1$ for $48 \mathrm{~h}$. After pretreatment with atorvastatin for 24 h, A549 cells had a partial transition to a fibroblast-like morphology. In addition, atorvastatin did not change the A549 cell shape after a 48 -h stimulation.
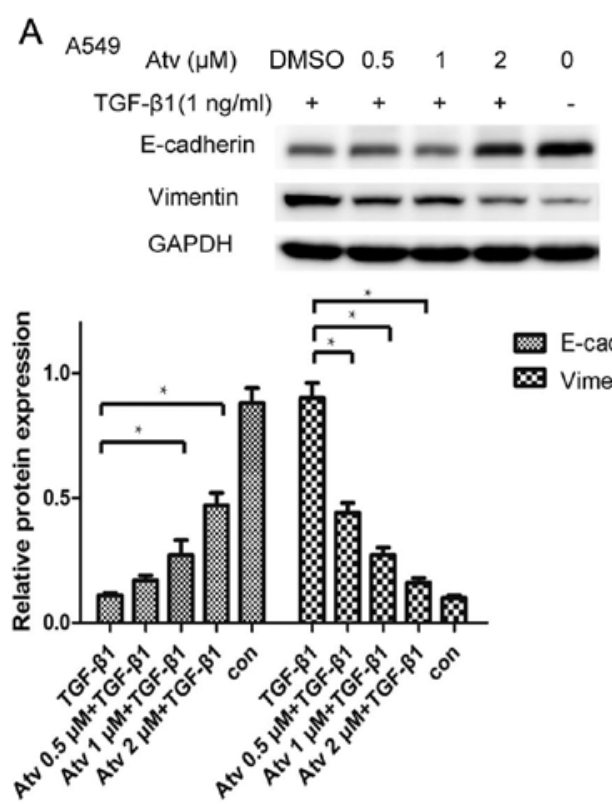

B
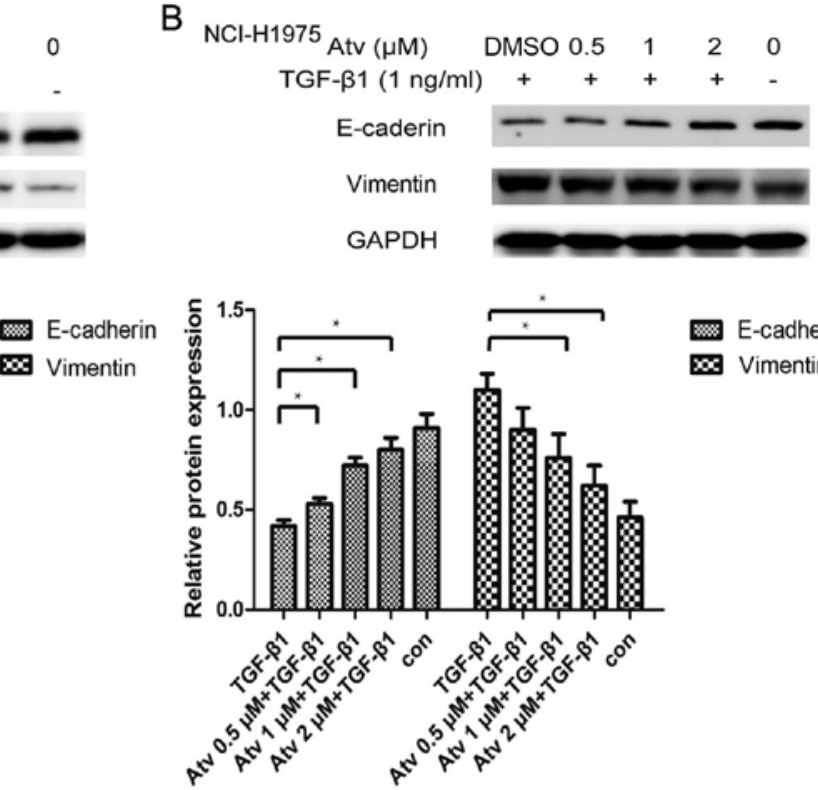

Figure 2. Expression of E-cadherin and vimentin was examined using western blot analysis to evaluate the EMT process. Densitometric analysis of E-cadherin and vimentin bands was performed and normalized with that of GAPDH. The bars indicate means \pm SD. The significance of the difference was assessed using the Student's t-test. "P $\leq 0.05$. (A) Atorvastatin (Atv) pretreatment effectively weakened TGF- $\beta 1$-stimulated downregulation of E-cadherin and upregulation of vimentin. (B) The results for the NCI-H1975 cells were the same as those for the A549 cells.

gene. Primer sequences of SphK1 were: F, 5'-CTGGCAGCTT CCTTGAACCAT-3' and R, 5'-TGTGCAGAGACAGCAGGT TCA-3'; the control primer sequences of GAPDH were: $F$, 5'-GGGAGCCAAAAGGGTCATCATCTC-3' and R, 5'-CCA TGCCAGTGAGCTTCCCGTTC-3'. The relative quantification of SphK1 mRNA was obtained by calculating $\Delta \Delta \mathrm{Ct}$.

Statistical analysis. Data are presented as mean \pm standard deviation (SD). SPSS 10.0 was used for statistic analysis and differences between groups were analyzed by the Student's t-test or ANOVA. Differences between groups were considered significant at $\mathrm{P}<0.05$.

\section{Results}

Atorvastatin partially inhibits the EMT process in A549 cells induced by TGF- $\beta 1$. A549 cells were serum-starved and treated with atorvastatin for $24 \mathrm{~h}$, and then exposed to atorvastatin and TGF- $\beta 1$ for another $48 \mathrm{~h}$. Cells displayed a complete transformation to fibroblast-like characteristics after $48 \mathrm{~h}$ with 

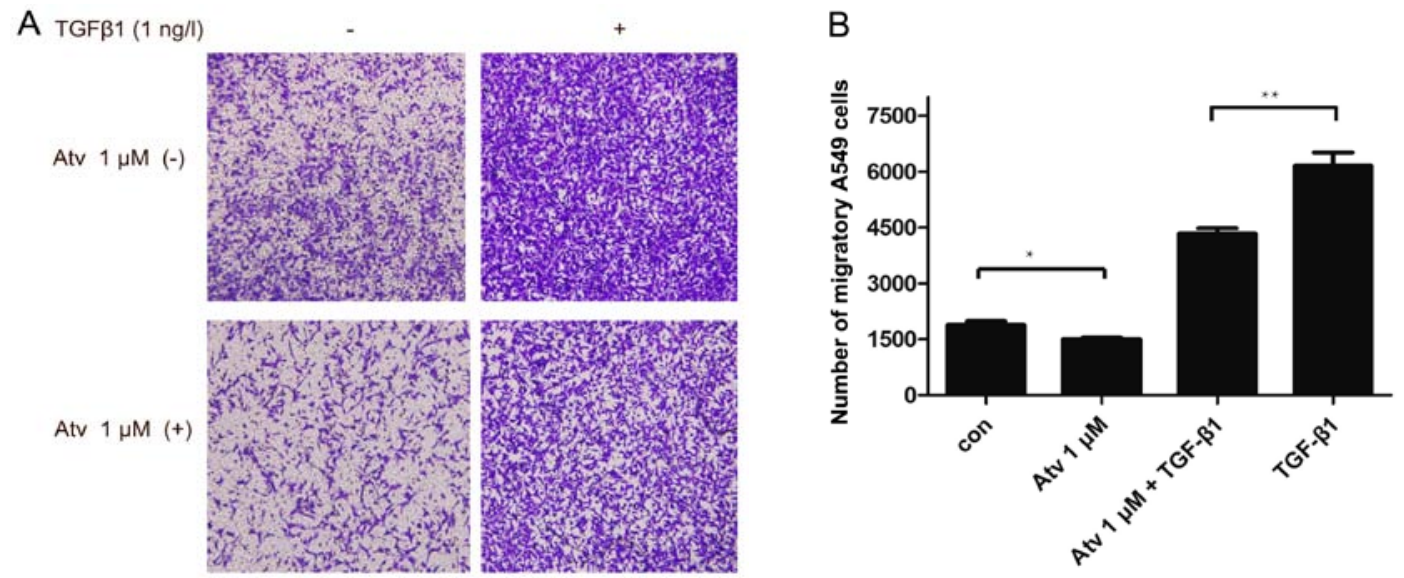

Figure 3. Atorvastatin inhibits the cell migration of A549 cells. Transwell assay was used to evaluate cell migration capability. (A) Representative images of A549 cells that migrated through the filters (magnification, $x 100$ ). (B) Quantification of the migrated cells. The bars indicated mean \pm SD. The significance of the difference was assessed using the Student's t-test; ${ }^{*} \mathrm{P} \leq 0.05,{ }^{* *} \mathrm{P} \leq 0.01$

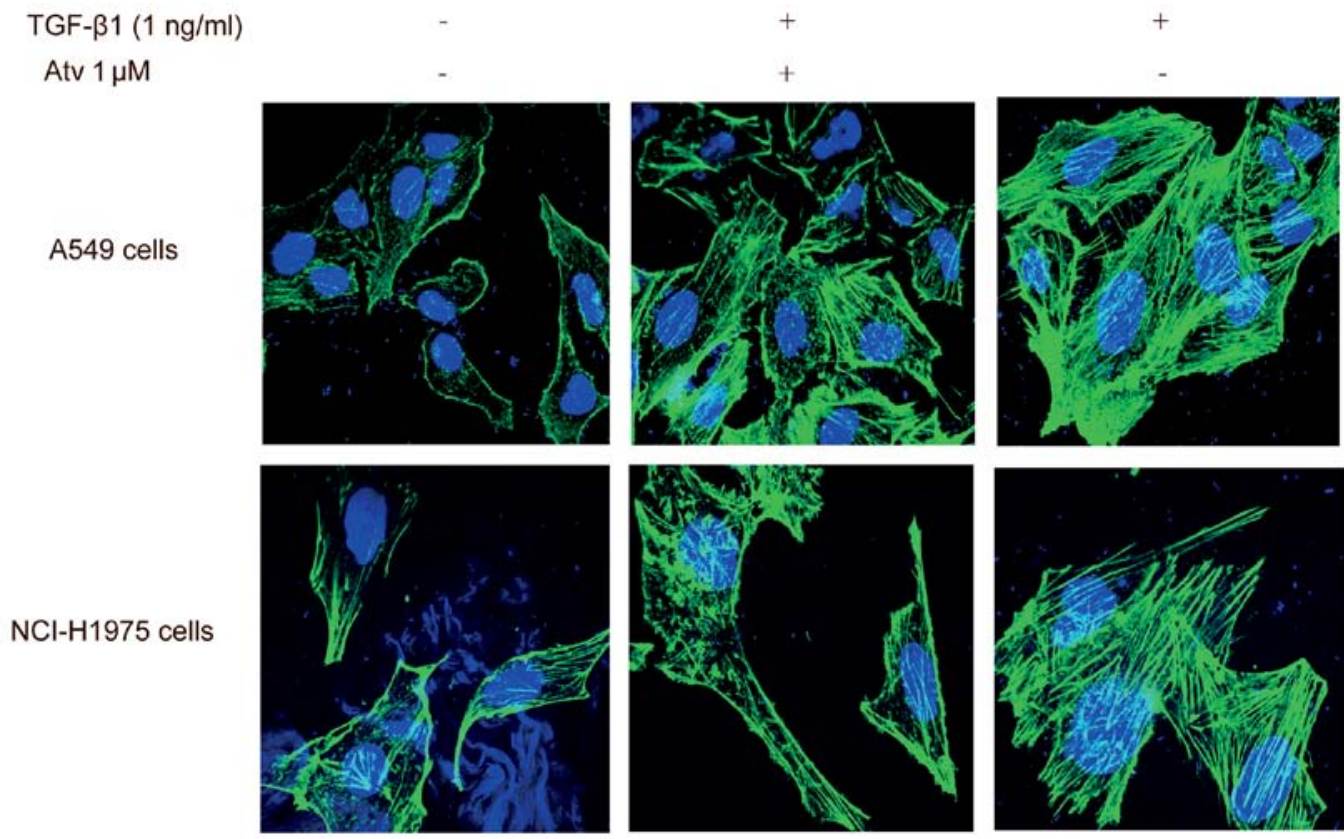

Figure 4. Actin fibers were detected using FITC-phalloidine by confocal laser scanning microscope. Following pretreatment with atorvastatin (Atv) for $24 \mathrm{~h}$, the actin fibers induced by TGF- $\beta 1$ in the A549 and NCI-H1975 cells were less and thinner than that noted in the positive control cells.

TGF- $\beta 1$ stimulation. However, when pretreated with atorvastatin for $24 \mathrm{~h}$, cells had a partial transition to a fibroblast-like morphology stimulated by TGF- $\beta 1$ for $48 \mathrm{~h}$. In addition, there was no recognizable change in cell shape after stimulation with atorvastatin alone for $48 \mathrm{~h}$ (Fig. 1). The result indicated that atorvastatin inhibited the TGF- $\beta 1$-stimulated morphological transition of the A549 cells to fibroblast-like cells.

The expression of E-cadherin and vimentin was not affected by treatment of various concentrations of atorvastatin. Atorvastatin pretreatment effectively weakened TGF- $\beta 1$-stimulated downregulation of E-cadherin as well as upregulation of vimentin. The alterations of EMT marker proteins such as E-cadherin and vimentin were further confirmed in the NCI-H1975 cells (Fig. 2). The result further confirmed that atorvastatin can partially inhibit the EMT process in A549 cells.
Atorvastatin inhibits cell migration and actin filament remodeling induced by TGF- $\beta 1$. It is known that EMT renders tumor cells with increased migratory potential (4). We evaluated the effect of atorvastatin on the elevated migration capabilities induced by TGF- $\beta 1$ in the A549 cells using Transwell assay. A549 cells were seeded on the upper chamber of the Transwell wells in the presence of TGF- $\beta 1$. A significant decrease in the number of A549 cells that migrated to the lower filters was observed when cells were pretreated with $1 \mu \mathrm{M}$ atorvastatin for $24 \mathrm{~h}$ (Fig. 3).

Remodeling of actin filaments is necessary for TGF- $\beta 1$ stimulated EMT. Actin filaments in epithelial cells, which are organized in cortical thin bundles, are bundled into thick contractile stress fibers after the EMT process (20). Following exposure to TGF- $\beta 1$ for $24 \mathrm{~h}$, atorvastatin pretreatment made the actin fibers of A549 cells less and thinner. This finding 


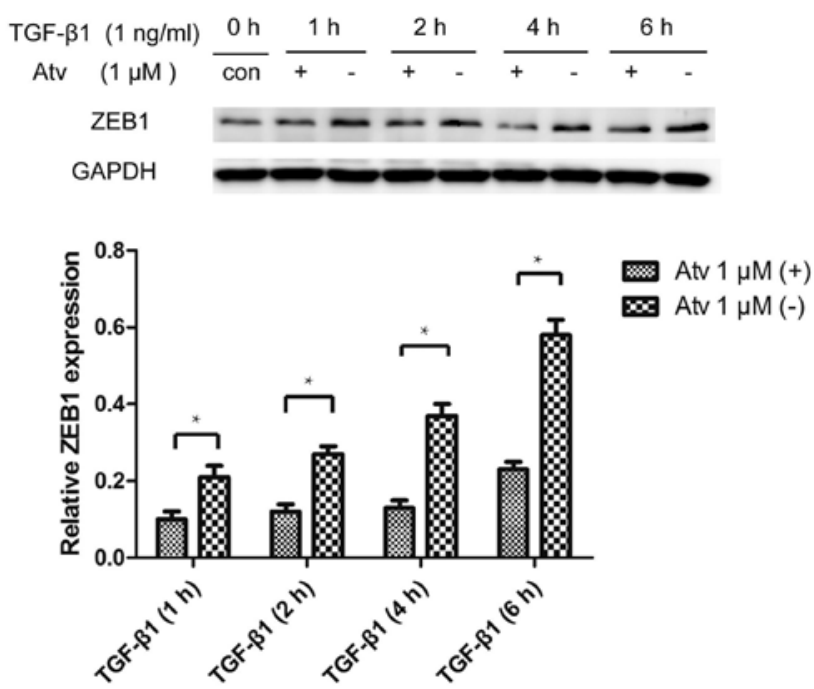

Figure 5. Atorvastatin (Atv) inhibits the expression of transcriptional factor ZEB1 in A549 cells. Western blotting was used to analyze the expression of ZEB1. In addition, densitometric analysis of ZEB1 bands was performed and normalized to that of GAPDH. The bars indicate mean \pm SD. The significance of the difference was assessed using the Student's t-test; ${ }^{*} \mathrm{P} \leq 0.05$.

was confirmed in the NCI-H1975 cells and the results indicate that atorvastatin can impede the actin filament remodeling process (Fig. 4).

Atorvastatin inhibits the TGF- $\beta 1$-stimulated upregulation of ZEB1. Numerous transcription factors which are essential for EMT, such as Snail, Slug and ZEB1, can repress genes important for maintaining epithelial polarity and organiza- tion (3). Our experiment found that atorvastatin inhibited the upregulation of ZEB1 induced by TGF- $\beta 1$. However, we did not find that Snail and Slug were inhibited by atorvastatin pretreatment (Fig. 5).

The activation of $\mathrm{Smad} 2 / 3$ by TGF- $\beta 1$ represents the canonical TGF- $\beta 1$ signaling system, and non-canonical signaling systems including MAPK-ERK1/2, PI3K-AKT and small GTP-binding proteins (21). In our research, atorvastatin did not inhibit the TGF- $\beta 1$-stimulated activation of Smad2/3, AKT and ERK1/2.

Atorvastatin attenuates the upregulation of SphK1 induced by $T G F-\beta 1$. It has been confirmed that TGF- $\beta 1$ upregulates SphK1 protein expression in A549 cells (18). In our experiment, we found that the upregulation of protein SphK1 induced by TGF- $\beta 1$ was also inhibited by atorvastatin pretreatment at $1 \mu \mathrm{M}$ (Fig. 6). However, at the mRNA level, real-time PCR found that atorvastatin did not inhibit the upregulation of SphK1 mRNA, which indicates that atorvastatin inhibits the translation of SphK1 at the post-transcriptional level when exposed to TGF- $\beta 1$ (Fig. 7).

Transient transfection of the SphK1 plasmid strengthens the EMT process induced by TGF- $\beta 1$ in the presence of atorvastatin. A549 cells were transiently transfected with the p-BABE SphK1 plasmid using Lipofectamine 2000. The EMT process induced by TGF- $\beta 1$ in the A549 cells, which were transfected with the SphK1 plasmid was strengthened. Expression of the EMT marker E-cadherin was lower in the cells transfected with the SphK1 plasmid. The result indicated that SphK1 transfection weakens the inhibitory effect of atorvastatin on the EMT process (Fig. 8).

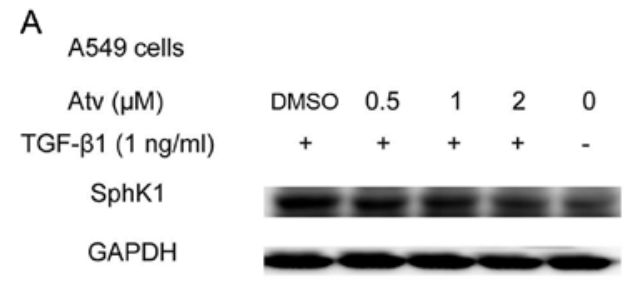

B
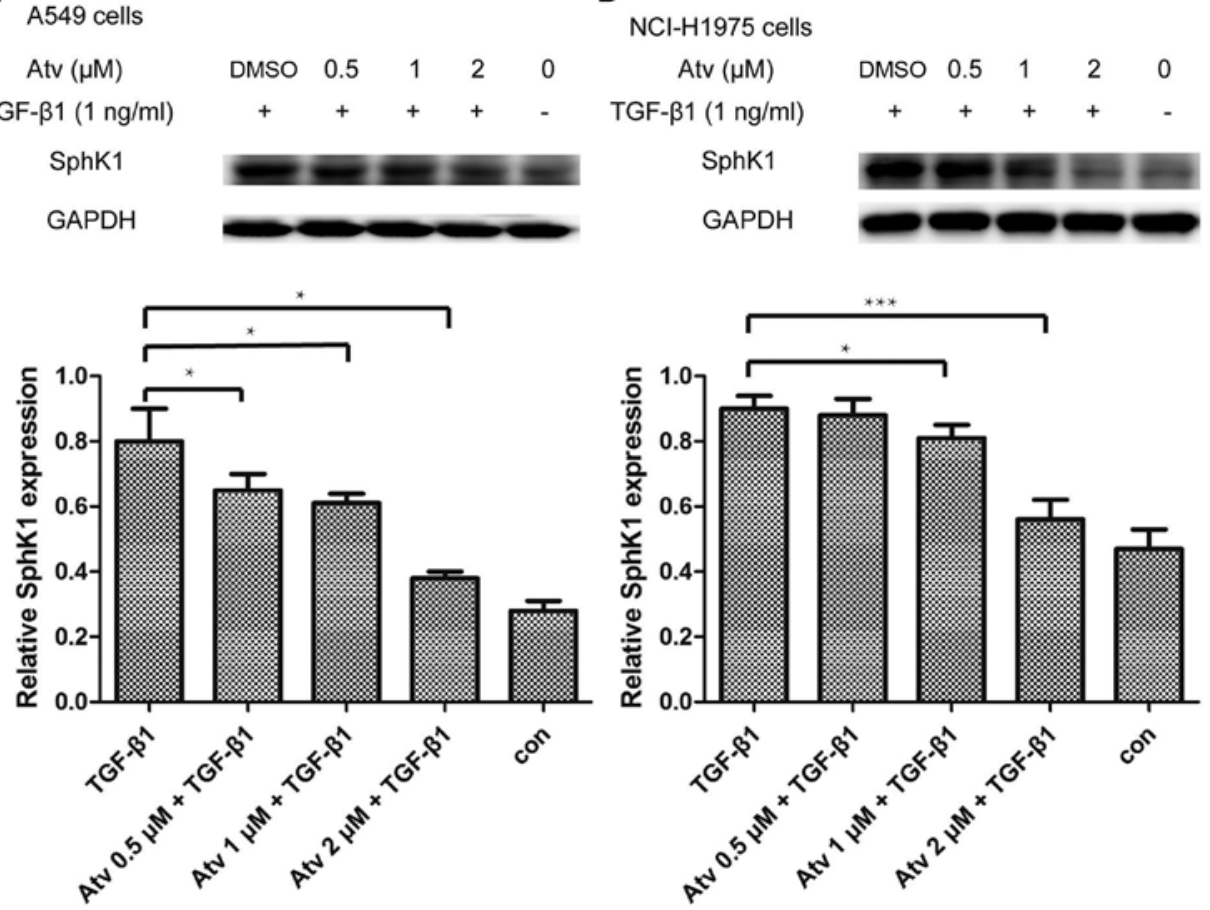

Figure 6. Atorvastatin (Atv) inhibits the upregulation of protein SphK1 in the A549 and NCI-H1975 cells. The expression of SphK1 was detected by western blot analysis. In addition, densitometric analysis of SphK1 bands was performed and normalized to that of GAPDH. The bars indicate mean \pm SD. The significance of the difference was assessed using the Student's t-test; "P $\leq 0.05$. (A) The results for the A549 cells. (B) The results for the NCI-H1975 cells. 


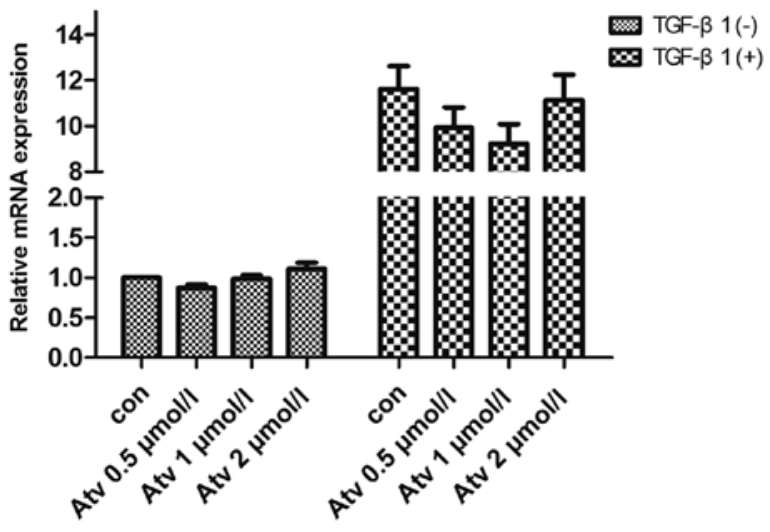

Figure 7. mRNA level of SphK1 was detected using real-time PCR assays with SYBR Premix Ex Taq ${ }^{\mathrm{TM}}$. Atorvastatin (Atv) did not affect the mRNA level of SphK1 with or without TGF- $\beta 1$ stimulation.

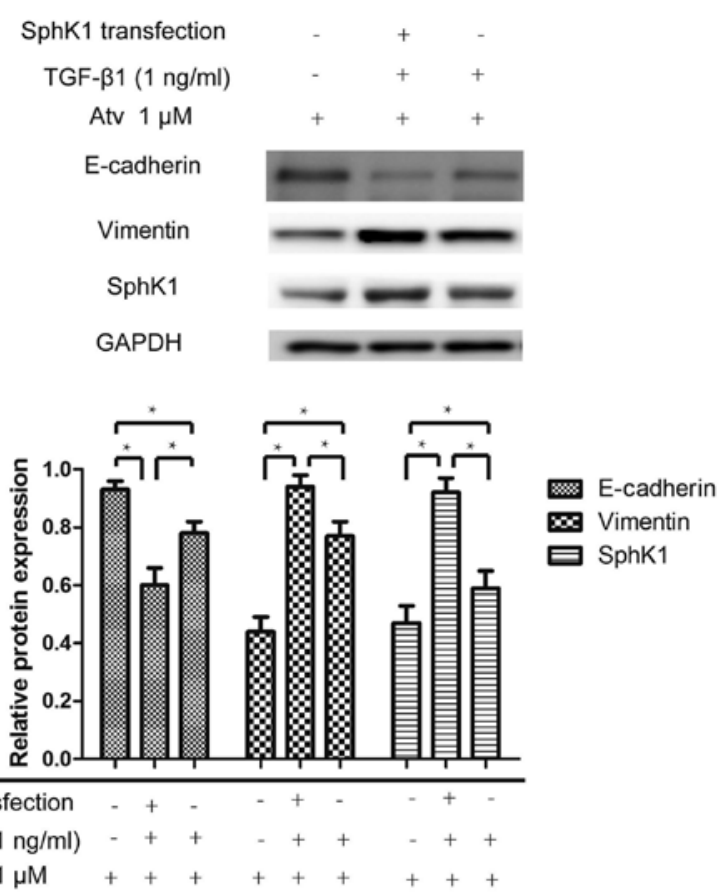

Figure 8. Transient transfection of the SphK1 plasmid strengthens the EMT process induced by TGF- $\beta 1$ in the presence of atorvastatin (Atv). A549 cells were transfected with pBABE-SphK1 plasmid and pBABE-puro using Lipofectamine 2000 reagent. The expression of E-cadherin, vimentin and SphK1 were detected by western blot analysis. In addition, densitometric analysis of E-cadherin, vimentin and SphK1 bands was performed and normalized to that of GAPDH. The bars indicated mean \pm SD. The significance of the difference was assessed using the Student's t-test; " $\mathrm{P} \leq 0.05$.

\section{Discussion}

Epithelial-to-mesenchymal transition (EMT) is an important mechanism for cancer metastasis. Recent research has confirmed that EMT is a potential biomarker of therapeutic resistance and is a potential drug target in breast cancer that warrants further investigation (22). Statins, HMG-CoA reductase inhibitors, are used extensively in the treatment of hyperlipidemia. Evidence shows that statins have anticancer effects. These secondary actions are known as pleiotropic effects. In the present study, we designed a series of experiments to evaluate the effect of atorvastatin on the EMT process induced by TGF- $\beta 1$ in NSCLC cells. MTT assay found that atorvastatin at $15 \mu \mathrm{M}$ significantly reduced the cell viability by $80 \%$. In addition, atorvastatin at $1-5 \mu \mathrm{M}$ did not affect the cell viability (23). In the present study, the cells were treated with atorvastatin at $0.5-2 \mu \mathrm{M}$, which had no effect on cell proliferation.

Differentiated epithelial cancer cells convert into migratory mesenchymal cancer cells via EMT, which may lead to cancer invasion, systemic cancer cell dissemination and metastasis. The metastatic potential is acquired by the loss of epithelial markers and the acquisition of mesenchymal markers. Our research found that atorvastatin treatment alone did not change the expression of the EMT markers, E-cadherin and vimentin, as well as cell morphology. However, after pretreatment with atorvastatin for $24 \mathrm{~h}$, the EMT process in A549 cells induced by TGF- $\beta 1$ was partially inhibited. The results indicated that atorvastatin can suppress the downregulation of E-cadherin and upregulation of vimentin induced by TGF- $\beta 1$, impede the formation of actin stress fibers and hinder cancer cell migration. The conversion of the cell shape into a fibroblast-like cell morphology was inhibited by atorvastatin pretreatment. These experiments demonstrated that atorvastatin can inhibit the EMT process induced by TGF- $\beta 1$ in vitro.

Transcription factors such as Snail, Slug and ZEB1 can induce EMT in cancer cells $(24,25)$. We further investigated whether these transcription factors are inhibited by atorvastatin during the EMT process. The results showed that atorvastatin impeded the upregulation of ZEB1 induced by TGF- $\beta 1$ and did not affect the expression of Snail and Slug.

These findings imply that atorvastatin may block the TGF- $\beta 1$ lower signal transduction in one manner or another. However, in our experiments, atorvastatin did not inhibit the activation of Smad2/3, ERK1/2 and AKT. Sphingosine kinase type 1 (SphK1), which catalyzes the phosphorylation of sphingosine to sphingosine-1-phosphate (S1P), has been shown to regulate various processes important for cancer progression (26). TGF- $\beta 1$ upregulates SphK1 in A549 cells and TGF- $\beta 1$-induced EMT in the A549 cells was inhibited by an antagonist of SphK1, providing evidence for crosstalk between SphK1 and TGF- $\beta 1$ (18). We found that atorvastatin pretreatment suppressed the upregulation of SphK1 protein induced by TGF- $\beta 1$, while the mRNA expression of SphK1 was not inhibited by atorvastatin as determined by the RT-PCR experiment. The results indicated that atorvastatin inhibited the transcription of SphK1 induced by TGF- $\beta 1$ stimulation. As S1P, catalyzed by SphK1 with sphingosine, can activate Smad2/3 in A549 cells (27), we considered that atorvastatin indirectly inhibits the activation of $S \operatorname{mad} 2 / 3$ by inhibiting the upregulation of SphK1 and downregulating the expression of transcription factor ZEB1.

When A549 cells were transiently transfected with the SphK1 plasmid, cells pretreated with atorvastatin had a lower expression of E-cadherin and a higher expression of vimentin compared with cells transfected with the SphK1-negative plasmid induced by TGF- $\beta 1$, indicating that the ability of atorvastatin to inhibit the EMT process was attenuated in cells highly expressing SphK1. This result further illustrated that atorvastatin inhibited the EMT process through the SphK1 pathway. 
In summary, the present study suggests that atorvastatin partially inhibits the EMT process in A549 cells induced by TGF- $\beta 1$ by attenuating the upregulation of SphK1. As EMT is one of the main mechanisms for the promotion of cancer invasion, and is a survival mechanism of cancer cells, statins may have beneficial effects for cancer patients in diverse ways.

\section{References}

1. Herbst RS, Heymach JV and Lippman SM: Lung cancer. N Engl J Med 359: 1367-1380, 2008.

2. Mehlen P and Puisieux A: Metastasis: A question of life or death Nat Rev Cancer 6: 449-458, 2006.

3. Tiwari N, Gheldof A, Tatari M and Christofori G: EMT as the ultimate survival mechanism of cancer cells. Semin Cancer Biol 22: 194-207, 2012.

4. De Craene B and Berx G: Regulatory networks defining EMT during cancer initiation and progression. Nat Rev Cancer 13: 97-110, 2013

5. Thiery JP, Acloque H, Huang RYJ and Nieto MA: Epithelialmesenchymal transitions in development and disease. Cell 139: 871-890, 2009.

6. Liao JK: Clinical implications for statin pleiotropy. Curr Opin Lipidol 16: 624-629, 2005.

7. Gazzerro P, Proto MC, Gangemi G, Malfitano AM, Ciaglia E, Pisanti S, Santoro A, Laezza C and Bifulco M: Pharmacological actions of statins: A critical appraisal in the management of cancer. Pharmacol Rev 64: 102-146, 2012.

8. Demierre MF, Higgins PD, Gruber SB, Hawk E and Lippman SM Statins and cancer prevention. Nat Rev Cancer 5: 930-942, 2005.

9. Menter DG, Ramsauer VP, Harirforoosh S, Chakraborty K, Yang P, Hsi L, Newman RA and Krishnan K: Differential effects of pravastatin and simvastatin on the growth of tumor cells from different organ sites. PLoS One 6: e28813, 2011.

10. Rao S, Porter DC, Chen X, Herliczek T, Lowe M and Keyomarsi K: Lovastatin-mediated $\mathrm{G}_{1}$ arrest is through inhibition of the proteasome, independent of hydroxymethyl glutaryl-CoA reductase. Proc Natl Acad Sci USA 96: 7797-7802, 1999.

11. Liu Y, Tang W, Wang J, Xie L, Li T, He Y, Deng Y, Peng Q, Li S and Qin X: Association between statin use and colorectal cancer risk: A meta-analysis of 42 studies. Cancer Causes Control 25: 237-249, 2014.

12. Khurana V, Bejjanki HR, Caldito G and Owens MW: Statins reduce the risk of lung cancer in humans: A large case-control study of US veterans. Chest 131: 1282-1288, 2007.

13. Shi M, Zheng H, Nie B, Gong W and Cui X: Statin use and risk of liver cancer: An update meta-analysis. BMJ Open 4: e005399, 2014.

14. Lustman A, Nakar S, Cohen AD and Vinker S: Statin use and incident prostate cancer risk: Does the statin brand matter? A population-based cohort study. Prostate Cancer Prostatic Dis 17: 6-9, 2014
15. Nielsen SF, Nordestgaard BG and Bojesen SE: Statin use and reduced cancer-related mortality. N Engl J Med 367: 1792-1802, 2012.

16. Baker DL, Pham TC and Sparks MA: Structure and catalytic function of sphingosine kinases: Analysis by site-directed mutagenesis and enzyme kinetics. Biochim Biophys Acta 1831: 139-146, 2013.

17. Kono Y, Nishiuma T, Nishimura Y, Kotani Y, Okada T, Nakamura $\mathrm{S}$ and Yokoyama M: Sphingosine kinase 1 regulates differentiation of human and mouse lung fibroblasts mediated by TGF-beta1. Am J Respir Cell Mol Biol 37: 395-404, 2007.

18. Milara J, Navarro R, Juan G, Peiró T, Serrano A, Ramón M, Morcillo E and Cortijo J: Sphingosine-1-phosphate is increased in patients with idiopathic pulmonary fibrosis and mediates epithelial to mesenchymal transition. Thorax 67: 147-156, 2012.

19. Ai J, Tang Q, Wu Y, Xu Y, Feng T, Zhou R, Chen Y, Gao X, Zhu Q, Yue X, et al: The role of polymeric immunoglobulin receptor in inflammation-induced tumor metastasis of human hepatocellular carcinoma. J Natl Cancer Inst 103: 1696-1712, 2011.

20. Haynes J, Srivastava J, Madson N, Wittmann T and Barber DL: Dynamic actin remodeling during epithelial-mesenchymal transition depends on increased moesin expression. Mol Biol Cell 22: 4750-4764, 2011.

21. Wendt MK, Allington TM and Schiemann WP: Mechanisms of the epithelial-mesenchymal transition by TGF-beta. Future Oncol 5: 1145-1168, 2009.

22. Yu M, Bardia A, Wittner BS, Stott SL, Smas ME, Ting DT, Isakoff SJ, Ciciliano JC, Wells MN, Shah AM, et al: Circulating breast tumor cells exhibit dynamic changes in epithelial and mesenchymal composition. Science 339: 580-584, 2013.

23. Miraglia E, Högberg J and Stenius U: Statins exhibit anticancer effects through modifications of the pAkt signaling pathway. Int J Oncol 40: 867-875, 2012.

24. Argast GM, Krueger JS, Thomson S, Sujka-Kwok I, Carey K, Silva S, O'Connor M, Mercado P, Mulford IJ, Young GD, et al: Inducible expression of TGF $\beta$, Snail and Zeb1 recapitulates EMT in vitro and in vivo in a NSCLC model. Clin Exp Metastasis 28: 593-614, 2011.

25. Alves CC, Carneiro F, Hoefler H and Becker KF: Role of the epithelial-mesenchymal transition regulator Slug in primary human cancers. Front Biosci 14: 3035-3050, 2009.

26. Shida D, Takabe K, Kapitonov D, Milstien S and Spiegel S: Targeting SphK1 as a new strategy against cancer. Curr Drug Targets 9: 662-673, 2008.

27. Xin C, Ren S, Kleuser B, Shabahang S, Eberhardt W, Radeke H, Schäfer-Korting M, Pfeilschifter J and Huwiler A: Sphingosine 1-phosphate cross-activates the Smad signaling cascade and mimics transforming growth factor-beta-induced cell responses. J Biol Chem 279: 35255-35262, 2004. 